\title{
El aprendizaje basado en problemas para mejorar el pensamiento crítico: revisión sistemática
}

\section{Problem based learning to improve critical thinking: a systematic review}

Jhon Bermúdez Mendieta

Universidad César Vallejo, Perú

jhbermen@hotmail.com

http://orcid.org/0000-0003-2390-4646

Recepción: 09/01/2021 | Aceptación: 19/04/2021 | Publicación: 10/05/2021

Cómo citar (APA, séptima edición):

Bermúdez Mendieta, J. (2021). El aprendizaje basado en problemas para mejorar el pensamiento crítico: revisión sistemática. Innova Research Journal, 6(2), 77-89.

https://doi.org/10.33890/innova.v6.n2.2021.1681

\section{Resumen}

Este estudio tuvo como propósito determinar cómo el uso de la metodología educativa Aprendizaje Basado en Problemas mejora el pensamiento crítico en estudiantes de secundaria. Para lograr lo propuesto, se desarrolló un análisis sistemático de la información, a través de la búsqueda de artículos científicos de acceso libre, en las bases de datos Dialnet, Scielo, Redalyc y Google académico. Como criterio de selección se consideró estudios empíricos que presenten resultados de intervenciones implementadas con Aprendizaje Basado en Problemas para mejorar el Pensamiento crítico, independiente de su género o idioma, publicados en los últimos seis años; artículos con intervención en muestras de estudiantes de educación secundaria. Los artículos seleccionados se organizaron en tablas para identificar sus objetivos, tipos de investigación y metodologías. Se destaca como resultado un mayor reporte de investigaciones realizadas en Asia. Se concluye que el Aprendizaje Basado en Problemas mejora significativamente el Pensamiento crítico en estudiantes de secundaria.

Palabras claves: aprendizaje basado en problemas; educación secundaria; pensamiento crítico.

\begin{abstract}
The purpose of this study was to determine how the use of Problem-Based Learning educational methodology improves critical thinking in high school students. To achieve this, a systematic analysis of the information was developed, through the search of scientific articles of free access,
\end{abstract}


in the databases Dialnet, Scielo, Redalyc and academic Google. As selection criteria, we considered empirical studies that present results of interventions implemented with Problem-Based Learning to improve critical thinking, independent of gender or language, published in the last six years; articles with intervention in samples of high school students. The selected articles were organized in tables to identify their objectives, types of research and methodologies. The result is an increased reporting of research conducted in Asia. It is concluded that Problem-Based Learning significantly improves critical thinking in high school students.

Keywords: problem-based learning; secondary education; critical thinking.

\section{Introducción}

El fenómeno de la globalización ha traído en estos últimos años grandes avances científicos y tecnológicos, los cuales exigen que el ser humano se encuentre preparado para enfrentar estos cambios, a través del desarrollo de capacidades y destrezas, las cuales deben ser introducidas desde los niveles básicos de la enseñanza con la finalidad de lograr una formación integral, con capacidades de pensamiento crítico; sin embargo se observa deficiencias en el pensamiento crítico en estudiantes de educación secundaria, y esto se ve reflejado en los reportes de evaluaciones tanto nacionales como internacionales donde aún muestran deficiencias en ciertas capacidades; por este motivo se viene a aplicando y fortaleciendo diversos métodos de enseñanza para mejorar esta habilidad en los estudiantes, ya que se observa que esta está directamente relacionada con el rendimiento académico.

La UNESCO (2017) en su agenda 2030, contempla que se debería asegurar que todos los niños en edad escolar tengan acceso gratuito a la educación básica; esta, además, debe ser equitativa y de calidad, con el objetivo de lograr un aprendizaje pertinente y efectivo. En tanto, (Botero et al., 2017, p. 88); (Nuñez Huamanta, 2020), afirman que el objetivo principal de la educación que se imparte a todos los niveles, no solo es brindar información de los diversos tópicos o especialidades, sino incentivar y estimular en el estudiante la aplicación de estrategias que sean complejas o de orden superior, entre estas se encuentra el pensamiento crítico. Por cuanto, (Ballesteros et al., 2018, p. 20), indican que para que el sistema educativo se mantenga a la vanguardia de este mundo globalizado, requiere de búsqueda continua de procesos de innovación y mejora, así como la aplicación de diversas estrategias que promuevan las habilidades cognitivas de orden superior. Asimismo, (Hidalgo y Murillo, 2017), refieren que, en la formación de los estudiantes, la evaluación es uno de los procesos que mejores resultados ha mostrado con respecto al aprendizaje y desarrollo, es por eso que en la actualidad existe una serie de evaluaciones estandarizadas. En tanto, (Bahamon y García, 2016), indican que el nivel educativo es uno de los principales indicadores para determinar el desarrollo de un país.

Por otro lado, (Sastoque et al., 2016, p. 148), afirman que el tener un buen desempeño en las labores, así como desarrollar las actividades con agrado, no es más que el resultado de las habilidades adquiridas en edades tempranas. Así mismo, (Moreno y Velásquez, 2017), indican que en la actualidad la sociedad requiere de personas con habilidades de pensamiento crítico, es por ello necesario que los sistemas educativos tengan como eje central, más allá de la mera transmisión de conocimientos, la aplicación de estrategias educativas que desarrollen procesos cognitivos que apunten al pensamiento de orden superior de los estudiantes. 
En tanto, la Evaluación PISA (2018), de 77 países evaluados, el Perú se ubica en el margen inferior; es decir, en el puesto 64. Según este reporte, el país obtuvo un promedio de 401 en lo que respecta a la compresión lectora; observándose una mejoría con respecto al resultado del año 2015 , que fue de 398. De igual manera se aprecia una mejoría en cuanto a las habilidades matemáticas, pues a diferencia del 2015 cuando se alcanzó 387 de promedio, el 2018 llegó a 400. Estos resultados son también alentadores en Ciencias, ya que, de 397 en el 2015, se alcanzó 404 unidades en el 2018. A nivel de Latinoamérica, el Perú aún se encuentra por debajo de sus pares de Chile, Brasil, Colombia y Argentina; sin embargo, desde el 2009, es el país que más ha crecido en las tres áreas evaluadas, observándose un incremento de +10.3 en lectura, +11.7 en matemática y +11.7 en ciencias. Del mismo modo, en las Evaluaciones de Logros de Aprendizaje (2019), en donde reporta que en el 2do de secundaria en el área de ciencia y tecnología: satisfactorio: 9.7\%, en proceso: $36.3 \%$, en inicio: $43.8 \%$, y previo al inicio: $10.1 \%$; en matemática: satisfactorio: $17.7 \%$, en proceso: $17.3 \%$, en inicio: $32.1 \%$, y previo al inicio: $33 \%$; y en lectura: satisfactorio: $14.5 \%$, en proceso: $25.8 \%$, en inicio: $42 \%$, y previo al inicio: $17.7 \%$. Si bien es cierto se observa discreta mejoría en los resultados de satisfactorio comparado con años previos, pero estos resultados son poco alentadores, ya que aún persiste un porcentaje alto de estudiantes que se encuentran en la fase de inicio y previo al inicio.

Ante estos resultados nace la inquietud de determinar si las metodologías innovadoras que se están utilizando en la enseñanza de la educación básica son las adecuadas para desarrollar el pensamiento crítico. Entre la metodología activa se encuentra el método aprendizaje basado en problemas (ABP), donde el aprendizaje se centra en el que aprende con una participación individual y colectiva siendo protagonistas de sus aprendizajes, se basa en el descubrimiento y el estudio guiado por los tutores. El ABP es uno de los métodos que favorece y ayuda a mejorar las habilidades, fortalecer sus capacidades, adquirir conocimiento, el concepto y la compresión del currículo escolar. Por consiguiente, es necesario que tanto en las instituciones educativas, así como las universidades se atienda situaciones de la vida cotidiana, situaciones reales, resolver problemas y atender necesidades, en tanto que esto se logrará enfrentándoles a situaciones problemáticas reales, utilizando metodologías de aprendizaje. A respecto, (Rodríguez Saenz, 2017), refiere que, de acuerdo con las nuevas innovaciones pedagógicas, la metodología del ABP es una herramienta activa que busca fortalecer pilares básicos como el aprender a aprender y el aprender a pensar.

Por lo expuesto, se plantea el problema de investigación ¿Cómo el aprendizaje basado en problemas mejora el pensamiento crítico en estudiantes de educación secundaria?, y para dar respuesta a este problema se plantea el objetivo: Determinar como el aprendizaje basado en problemas mejora el pensamiento crítico en estudiantes de educación secundaria. Así mismo, ésta investigación se justifica porque busca demostrar cuán importante es aplicar el ABP para potenciar el pensamiento crítico en estudiantes de educación secundaria, además este estudio tiene la relevancia en el campo de la didáctica, ya que el desarrollo de habilidades de esta naturaleza es esencial para lograr estudiantes críticos y reflexivos.

\section{Metodología}

Para realizar el presente artículo se procedió a realizar una revisión sistémica, la población lo constituyen todos los artículos científicos de revistas indexadas, de las bases de datos de 
ResearchGate, Scielo, Semantic Scholar, Redib, Scielo, Google académico, ERIC, Dialnet, IOPscience, Redalyc, etc. cuya temática se corresponde con el uso de la metodología educativa Aprendizaje Basado en Problemas y el desarrollo de pensamiento crítico en estudiantes de secundaria, publicados desde el año 2015 hasta el 2020, cuyo criterio de inclusión son: (a) estudios empíricos que presentaran resultados de intervenciones implementadas con ABP para mejorar el pensamiento crítico, independiente de su género o idioma (b) artículos con intervención en muestras de estudiantes de educación secundaria, y (c) estudios donde sus participantes no posean alguna necesidad educativa especial o superdotados.

Para el desarrollo de esta revisión sistémica se siguió las recomendaciones de la de la Declaración Prisma (Urrutia y Bonfill, 2010), es una herramienta fue concebida para contribuir a mejorar la calidad y la transparencia en la publicación de las revisiones sistemáticas, el cual inicia con la identificación de la información, continúa por el número total de registros o citas únicas una vez eliminado los duplicados y termina con los estudios individuales incluidos en la revisión sistemática.

\section{Figura 1}

Diagrama de flujo - Declaración Prisma

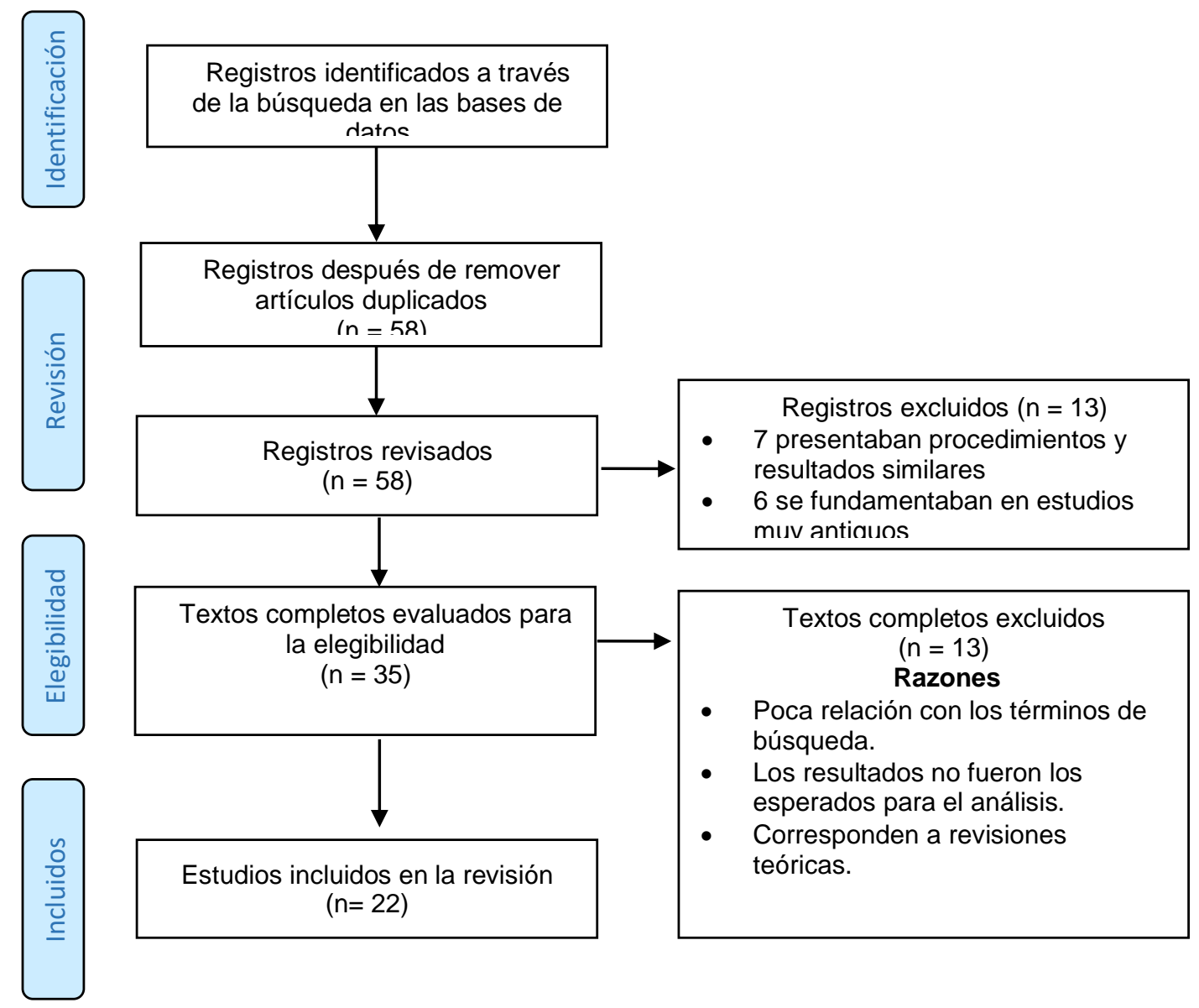


Se detalla cada uno de los pasos: en primer lugar, es la identificación de los estudios referidos a las variables en análisis; se consultó desde los meses de abril a octubre del 2020 las bases de datos: ResearchGate, Scielo, Semantic Scholar, Redib, Scielo, Google académico, ERIC, Dialnet, IOPscience, Redalyc, etc. Publicados desde el 2015 hasta el 2020, cuya temática se corresponde con el uso de la metodología educativa Aprendizaje Basado en Problemas y el desarrollo de pensamiento crítico en estudiantes de secundaria. En la opción búsqueda de las bases de datos, se introdujo palabras claves en español e inglés como: aprendizaje basado en problemas escritura (Problem-based learning), pensamiento crítico (critical thinking), educación secundaria (secondary education), estos términos fueron combinados con operadores booleanos como o (OR) e y (AND). También se depuró la búsqueda aplicando la opción "filtro" por años, tipo de publicación y acceso libre. Selección de los datos producidos por estos estudios que forman parte de la muestra. Los artículos que se obtuvieron de las bases de datos fueron analizados de manera preliminar a partir del título, resumen y metodología, los preseleccionados $(n=58)$ pasaron a una tabla donde se aplicó los criterios de inclusión y exclusión para ser parte de esta investigación. Finalmente, se seleccionaron 22 artículos. Análisis comparativo de los datos comunes y en conjunto. Los artículos seleccionados pasaron a una tabla donde se consignó información significativa para esta revisión como objetivos, metodología y resultados pre y post intervención. Estos datos fueron sometidos al análisis comparativo y crítico. Al hacer el análisis, se permitió tener una percepción global y particular sobre los alcances y efectividad que tuvieron las intervenciones en la producción de textos escritos, lo que llevó a la interpretación y elaboración de las conclusiones.

Para el análisis de datos los artículos de este estudio fueron disgregados en categorías mínimas y distribuidas en tablas estructuradas para el análisis comparativo y reflexivo sobre los alcances de las intervenciones. No se dio un tratamiento estadístico puesto que no se pretendió llegar al metaanálisis.

\section{Resultados}

El 100\% de los artículos en revisión son originales; el 90\% de las publicaciones científicas revisadas fueron realizadas por varios investigadores. El 85\% de los artículos fueron publicados en revistas de educación, un $13 \%$ fueron publicados en revistas de investigación científica y tecnológica, y solamente el $2 \%$ fueron publicados en revistas que corresponde al área de investigación médica. El 55\% de los artículos se encuentran en el idioma inglés, el 38\% de las publicaciones se encuentran en español, el 5\% de las publicaciones se encuentran en indonesio y un $2 \%$ de las publicaciones se encuentran en portugués.

Al clasificar estas publicaciones de acuerdo al espacio geográfico tenemos, que el $59 \%$ de estas publicaciones científicas fueron realizadas en Asia, el 34\% de las publicaciones corresponden a América, el $6 \%$ fueron realizados en Europa y solo un $2 \%$ de las publicaciones corresponden a África. Los artículos revisados fueron publicados en los últimos 6 años, de los cuales se observa que el 3\% de las publicaciones corresponden al año 2020, el 16\% corresponden al año 2019, el $22 \%$ corresponden al año 2018, el 28\% corresponden al año 2017, el 19\% corresponden al año 2016 y solo un $12 \%$ de las publicaciones corresponden al año 2015. 
Al revisar la selección de artículos científicos, según criterios establecidos, se observa que el 78\% de los artículos reportan un tipo y diseño de investigación que se corresponde al interés para la revisión (experimentales). El 42\% de los artículos lo denominan las variables igual que las del estudio y el $100 \%$ de las revisiones contienen de cierta forma la variable en estudios. Así mismo, se encontró que el $98 \%$ de los estudios revisados presentan una técnica que contribuye a demostrar el planteamiento de la investigación. Además, el 93\% de artículos analizados utilizaron herramientas adecuadas. También se encontró que el método utilizado contribuye a demostrar el planteamiento del estudio. Así mismo, las estrategias de intervención favorecen y refuerzan la hipótesis. Finalmente, luego de analizar diversos aspectos de estos artículos se concluye que solamente el $38 \%$ cumplen con los requisitos formulados que vienen a ser 22 artículos que cumplieron con criterios.

Así mismo, los tipos de diseño de investigación se clasificaron siguiendo a (Hernández et al., 2014, p. 89), encontrándose que el 86\% corresponden a estudios cuasi experimentales y el 14\% fueron de tipo pre experimental. De este modo, los diseños adoptados en el 100\% de las investigaciones se inclinan por un trabajo empírico-analítico de la información para precisar la efectividad de las intervenciones en la variable dependiente.

Con respecto a la técnica de recojo de información, estas fueron coherentes con el instrumento que se utilizó en la investigación, la totalidad de ellas se centraron en la técnica de la observación. Sobre los instrumentos, se observa con mayor prevalencia a los el pre test y pos test en un $50 \%$, seguido de cuestionarios y rúbricas. Para el reporte de información sobre si hubo mejoras entre el pre test y el post test, utilizaron técnicas estadísticas como la prueba t-student, ANCOVA, o Wilcoxon para obtener resultados en porcentajes o promedios. Con respecto a la población y muestra, se observa que solamente un 55\% especifican el tamaño de la muestra y el $100 \%$ de las investigaciones corresponde al nivel secundaria. Además, se observa que el 100\% de las investigaciones tuvieron como objetivo común el probar la efectividad del aprendizaje basado en problemas para mejorar el pensamiento crítico en estudiantes de secundaria.

La teoría en la cual se sustenta esta investigación con respecto al aprendizaje basado en problemas es el constructivismo, aunque no esté explícitamente en algunas investigaciones. Con respecto a las estrategias tenemos que el $100 \%$ de los artículos revisados se basan en programa de estudio y con respecto a la duración o sesiones que se realicen en el programa, tenemos que solamente el $32 \%$ especifican el número de sesiones o el tiempo que duró el programa. Así mismo al analizar el pre tratamiento y post tratamiento, se encuentra que solamente el $32 \%$ de los artículos revisados especifican los resultados pre tratamiento.

\section{Discusión}

Tenemos estudios tanto pre experimentales, estudios mixtos y cuasi experimentales que apoyan la hipótesis de que el aprendizaje basado en problemas mejora el pensamiento crítico.

Entre los estudios pre experimentales, tenemos a (Afdareza et al., 2020, p.283), quienes utilizaron el modelo de Plomp y encontraron que, al aplicar su instrumento en forma de dispositivo con la metodología educativa en estudio, había un aumento en la capacidad de pensamiento crítico 
de los estudiantes. Así mismo tenemos a (Haridza y Irving, 2017), quienes afirman que el modelo de aprendizaje basado en problemas de 4 áreas básicas (PBL4C) es un enfoque de aprendizaje que desarrolla significativamente las habilidades de pensamiento crítico de los estudiantes. En tanto, (Yuliati et al., 2018, p. 5), concluyen que la auténtica implementación del ABP sobre el aprendizaje de la física en las escuelas secundarias públicas puede mejorar las habilidades de pensamiento crítico de los estudiantes. Además (Safitri et al.,2018, p. 255), encuentran correlación positiva entre el logro cognitivo con las habilidades de pensamiento crítico.

Entre los estudios mixtos tenemos a (Jailani et al., 2017, p. 256), encontraron que la implementación del ABP ha sido más efectiva que la implementación del aprendizaje expositivo en términos de mejorar el habilidad de pensamiento de orden superior (HOTS)de los estudiantes; sin embargo, la implementación del ABP no ha sido más efectiva que el aprendizaje expositivo en términos de mejorar el carácter de los estudiantes, y esto obedece a varios factores en los cuales se sospechan es que los estudiantes no están acostumbrados a asistir al proceso de aprendizaje basado en problemas, conllevando a que las actividades de colaboración grupal no han sido implementado de manera efectiva, la responsabilidad, el cuidado y la cooperación, hayan sido menos entrenados; así mismo, a pesar de haberse realizado una serie de intervenciones de los docentes dentro del proceso de aprendizaje para mejorar el carácter de los estudiantes, es decir, la independencia, la perseverancia y el trabajo duro, no se hayan formado al máximo; y la baja capacidad de resolución de problemas de los estudiantes ha resultado en su falta de confianza en sí mismos. Así mismo, (Ahamad et al., 2017, p. 11), encontraron que el ABP influye positivamente en el rendimiento de los estudiantes en el aprendizaje de la geometría.

Entre los estudios cuasi experimentales en las cuales se observan que el ABP mejora el aprendizaje, tenemos a (Suryawati et al., 2020, p. 176), estudio con pre test y post test, encontraron que al aplicar la metodología en estudio produce un efecto significativo en el aprendizaje. Así mismo, (Colón y Ortiz, 2020), demostraron que el uso del ABP produce un efecto significativo en la mejora de las destrezas de comprensión y análisis. En tanto, (Casa et al.,2019, p. 119); (Malmia et al., 2019, p. 1141), utilizando diseño de grupo control solo post prueba, al aplicar la estrategia $\mathrm{ABP}$, observaron que mejoró significativamente el rendimiento académico de los estudiantes, debido a que existe un ascenso significativo en el desarrollo de las sesiones y en la prueba. En tanto (Leiva Sánchez, 2016) y (Widyatiningtyas et al., 2015, p. 114), encontraron que el ABP mejora la capacidad para resolver problemas matemáticos comparado con el aprendizaje convencional. Además, (Mohd et al., 2016, p. 59), demostró que, mediante una planificación y preparación adecuada, las actividades del ABP pueden aumentar eficazmente el compromiso de los estudiantes y mejorar su comprensión de los conocimientos de contenido geográfico.

Entre los estudios cuasi experimental en las cuales se observa que el ABP mejora el pensamiento crítico, comparado con la metodología tradicional de enseñanza, tenemos a (Daud y Isana, 2019); (Santyasa et al., 2019, p. 640); (Aswan et al., 2019, p. 4); (Alfaro Luján, 2019); (Herzon et al., 2018, p. 45); (Sapallanay Baltazar, 2016) y (Jácome Maguiña, 2015), los cuales inciden que el ABP mejora las habilidades de pensamiento crítico. En tanto (Ramdiah et al. 2018, p. 31), demostró que el modelo ABP tuvo un efecto positivo en HOTS. Así mismo, (Bashith et al., 2017, p. 100), indica que el modelo de aprendizaje basado en problemas afecta las habilidades de pensamiento crítico. En tanto, (Anazifa y Djukri, 2017), afirman que el aprendizaje basado en proyectos y el aprendizaje basado en problemas afectan la creatividad y el pensamiento crítico del 
estudiante. Así mismo (Anazifa R, 2016), demostró que el Aprendizaje Basado en Problemas afecta al pensamiento crítico, afecta al logro de los estudiantes en aspectos cognitivos, además encontró relación significativa entre el pensamiento crítico y el logro de los estudiantes en aspectos cognitivos.

Entre los estudios cuasi experimentales que observan el ABP mejora el rendimiento académico y el pensamiento crítico, tenemos a (Mundilarto y Helmiyanto, 2017), refieren que el modelo ABP produce efecto positivo en el rendimiento del aprendizaje y la habilidad de pensamiento crítico de los estudiantes. Así mismo (Nisa et al. 2017, p. 39), concluyeron que los estudiantes que recibieron tratamiento, obtuvieron mejores resultados que los estudiantes del grupo control en las pruebas de capacidad de resolución de problemas y en la prueba de rendimiento y fueron mejores en sus habilidades de pensamiento crítico. En tanto, (Wahyu et al., 2017, p. 637), encontraron que en la prueba de hipótesis la ganancia media de los estudiantes en el pensamiento crítico y la capacidad de resolución de problemas enseñada por el aprendizaje basado en problemas era mejor que el aprendizaje convencional. Además, (Yanti y Indra, 2017), encontraron que el promedio de habilidades de pensamiento crítico matemático entre los estudiantes que obtienen el aprendizaje de las matemáticas usando el modelo de aprendizaje basado en problemas es más alto que los estudiantes que obtienen el aprendizaje de las matemáticas usando el modelo de investigación guiada. Así también, (Aidoo et al2016, p. 106), en su estudio de 2 grupos, al grupo control se le enseñó con el método de lectura tradicional, mientras que el grupo experimental recibió instrucción con $\mathrm{ABP}$, concluyeron que el $\mathrm{ABP}$ es una forma eficaz de enseñar química a fin de mejorar el pensamiento crítico y las habilidades de resolución de problemas de los estudiantes.

También tenemos a (Rahmad et al., 2016, p. 37), encontraron que hay un efecto del modelo de aprendizaje basado en problemas en las habilidades de pensamiento crítico de los estudiantes en la enseñanza sobre el ecosistema y los materiales ambientales. En tanto, (Villalobos et al. 2016, p. 578), encontraron que la aplicación del ABP favorece el desarrollo del pensamiento crítico, además observaron que los estudiantes formados con ABP lograron un mayor desarrollo de habilidades como la evaluación y autorregulación al compararse con el método de enseñanza tradicional. Así mismo, (Lestari et al., 2015, p. 470), determinaron que el ABP mejora las habilidades de pensamiento crítico y actitudes sociales de los estudiantes. Por otro lado, también hay investigaciones correlaciones como la de (Correa et al., 2019, p. 73), en donde encuentran relación entre metacognición y motivación a pensar críticamente, lo cual ratifican la necesidad de considerar estos elementos cognitivo emocionales en el fomento del pensamiento crítico.

Además, existen estudios en otros niveles académicos como la de (Mulyanto et al., 2018, p. 39), estudio realizado en alumnos de quinto grado de primaria, encontraron diferencias significativas en los resultados del aprendizaje de las matemáticas entre los estudiantes que siguen el modelo de aprendizaje basado en problemas con el modelo convencional, además encontraron diferencia significativa entre los estudiantes que tienen una capacidad de pensamiento crítico alta y baja. Sin embargo, no encontraron efecto de interacción entre el modelo de aprendizaje y las habilidades de pensamiento crítico.

Así mismo se observa que de la literatura revisada, existe una amplia información sobre la variable aprendizaje basado en problemas tanto de formación básica, lo cual incluye tanto 
educación primaria y secundaria, también observamos que hay múltiples estudios en el ámbito superior tanto técnico como universitario en las diversas disciplinas de formación, además se observa resultados o efectos similares sobre los aspectos en estudio como mejora de habilidades de pensamiento crítico, mejora en el aprendizaje y rendimiento académico, desarrollo de destrezas de comprensión y análisis en las diversas las diversas áreas o disciplinas de estudio.

Es así que, se observa en todos los artículos revisados en el presente estudio, presenta efectos beneficiosos del ABP en el aspecto que mejora el rendimiento académico, mejora las actitudes sociales y mejora el pensamiento crítico de los estudiantes.

\section{Conclusiones}

El Aprendizaje Basado en Problemas que en un inicio se creó en las universidades, se ha visto que, al aplicarse en el nivel secundario, produce mejoras en el pensamiento crítico.

Las publicaciones científicas comprendidas en esta revisión sistemática, solo reportaron resultados de intervenciones empíricas en el nivel secundaria, obviándose las publicaciones de otros niveles educativos.

Todas las investigaciones reportaron incrementos en los porcentajes o puntajes promedios sobre el Pensamiento crítico como efecto del Aprendizaje basado en problemas, independiente de la duración de la aplicación programa.

Estos resultados ofrecieron la posibilidad de tener una visión amplia sobre la aplicación de las metodologías educativas y su efecto.

Después de haber desarrollado la presente investigación, se recomienda:

A las autoridades educativas, incentivar el uso de metodologías educativas como el Aprendizaje basado en problemas con el objetivo de mejorar el pensamiento crítico de los estudiantes, ya que su efectividad está científicamente documentada.

Capacitar a los docentes, en el uso adecuado de las metodologías educativas como el Aprendizaje basado en problemas, para lograr de manera eficiente buenos resultados.

A los investigadores de la educación, ampliar estudios de esta índole en todos los niveles educativos, así como procurar la publicación en revistas indexadas.

\section{Referencias Bibliográficas}

Afdareza, M., Yuanita, P., \& Maimunah. (2020). Development of Learning Device Based on 21st CenturySkill with Implementation of Problem Based Learning to Increase Critical Thinking Skill of Students on Polyhedron for Grade 8th Junior High School. Journal of Educational Sciences, 4(2), 273-284. http://dx.doi.org/10.31258/jes.4.2.p.273-284 
Ahamad, S.N.S.H., Li, H.C., Shahrill, M., \& Prahmana, R.C.I. (2018). Implementation of problem-based learning in geometry lessons. Journal of Physics: Conference Series, 943(1), 012008. https://doi.org/10.1088/1742-6596/943/1/012008.

Aidoo, B., Kwadwo Boateng, S., Siaw Kissi, P., \& Ofori, I. (2016). Effect of Problem-Based Learning on Students' Achievement in Chemistry. Journal of Education and Practice, 7(33), 103-108.

Alfaro Luján, S. (2019). Programa didáctico centrado en estudio de casos y el desarrollo del pensamiento crítico de las estudiantes de segundo de secundaria [Tesis de doctorado, Universidad Nacional de Trujillo]. http://dspace.unitru.edu.pe/handle/UNITRU/15843

Anazifa, R. (2016). (16-17 de mayo de 2016). The Effect of Problem-Based Learning on Critical Thinking Skills and Student Achievement [Discurso principal]. Proceeding of $3 \mathrm{Rd}$ international conference on research, Implementation and education of mathematics and science, Yogyakarta.

Anazifa, R., \& Djukri. (2017). Project-based learning and problem- based learning: are they effective to improve student's thinking skills? Jornal Pendidikan IPA Indonesia, 6(2), 346355. https://doi.org/10.15294/jpii.v6i2.11100

Astriani, N., Surya, E., \& Syahputra, E. (2017). The effect of problem based learning to students' mathematical problem solving ability. International Journal of Advance Research and Innovative Ideas in Education, 3(2), 3441-3446.

Aswan, D., Lufri, L., \& Sumarmin, R. (5-6 October 2017). Influence of Problem Based Learning on Critical Thinking Skills and Competence Class VIII SMPN 1 Gunuang Omeh. [Discurso principal]. IOP Conference Series: Materials Science and Engineering, Padang, West Sumatera, Indonesia

Bahamon Correa, E., y García Velandia, A. (2016). Revisión de casos de modelos educativos a nivel mundial [Tesis de grado, Universidad Piloto de Colombia]. http://repository.unipiloto.edu.co/handle/20.500.12277/2428

Ballesteros Delgadillo, D., Castro Garzón, G., y Torres Páez, J. (2018). El Aprendizaje Basado en Problemas como estrategia para desarrollar habilidades de pensamiento crítico: Situaciones del uso de la lengua extranjera inglés en un contexto real. [Tesis de maestría, Universitaria Uniagustiniana]. http://repositorio.uniagustiniana.edu.co/handle/123456789/701

Bashith, A., \& Amin, S. (2017). The Effect of Problem Based Learning on EFL Students' Critical Thinking Skill and Learning Outcome. Al-Ta Lim Journal, 24(2), 93-102. https://doi.org/10.22219/jpbi.v4i1.5490

Botero Carvajal, A., Alarcón, D., Palomino Angarita, D., y Jiménez Urrego, A. (2017). Pensamiento crítico, metacognición y aspectos motivacionales: una educación de calidad. Poiésis, 33, 85-103. https://doi.org/10.21501/16920945.2499

Casa Coila, M., Huatta Pancca, S., y Mancha Pineda, E. (2019). Aprendizaje Basado en Problemas como estrategia para el desarrollo de competencias en estudiantes de educación secundaria. Revista de Investigación en Comunicación y Desarrollo, 10(2), 111-121. https://doi.org/10.33595/2226-1478.10.2.383

Colón Ortiz, L., y Ortiz Vega, J. (2020). Efecto del Uso de la Estrategia de Enseñanza Aprendizaje Basado en Problemas (ABP) en el Desarrollo de las Destrezas de Comprensión y Análisis de la Estadística Descriptiva. Revista Iberoamericana de Evaluación Educativa, 13(1), 205-223 https://doi.org/10.15366/riee2020.13.1.009 
Correa Gacitúa, J., Ossa Cornejo, C., y Sanhueza Morales, P. (2019). Sesgo en razonamiento, metacognicion y motivación al pensamiento crítico en estudiantes de primer año medio de un establecimiento de Chillán. Revista de Estudios y Experiencias en Educación, 18(37), 61-77. https://doi.org/10.21703/rexe.20191837correa8

Daud, D., \& Isana Supiah, Y. (2019). The effect of problem based learning model on critical thinking skills in the context of chemical reaction rate. Journal of Education and Learning, 13(3). 395-401. https://doi.org/10.11591/edulearn.v13i3.13887

Eviyanti, C., Surya, E., \& Syahputra, E. (2017). Improving the Students' Mathematical Problem Solving Ability by Applying Problem Based Learning Model in VII Grade at SMPN 1 Banda Aceh Indonesia. International Journal of Novel Research in Education and Learning, 4(2), 138-144. https://www.researchgate.net/publication/318529138

Haridza, R., \& Irving, K. (2017). Developing Critical Thinking of Middle School Students using Problem Based Learning 4 Core Areas (PBL4C) Model. IOP Conf. Series: Journal of Physics. https://doi.org/10.1088/1742-6596/812/1/012081

Hernández Sampieri, R., Fernández Collado, C., y Baptista Lucio, M. d. (2014). Metodología de la Investigación (6 ed.). México: Mc Graw Hill.

Herzon, H., Budijanto, \& Utomo, D. (2018). Pengaruh Problem-Based Learning (PBL) terhadap Keterampilan Berpikir Kritis. Jurnal Pendidikan: Teori, Penelitian, dan Pengembangan, 3(1), 42-46. http://dx.doi.org/10.17977/jptpp.v3i1.10446

Hidalgo Farran, N., y Murillo Torrecilla, F. (2017). Las Concepciones sobre el Proceso de Evaluación del Aprendizaje de los Estudiantes. Revista Iberoamericana sobre Calidad,

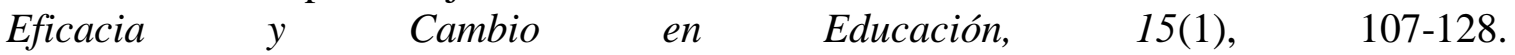
https://doi.org/10.15366/reice2017.15.1.007

Jácome Maguiña, Y. (2015). Aprendizaje Basado en Problemas para desarrollar el Pensamiento Crítico en el Área de Ciencias Sociales en los estudiantes del Tercer Grado de secundaria de la I.E. "Pedro Pablo Atusparia" de Vicos, Carhuaz 2014 [Tesis de maestría, Universidad Nacional Pedro Ruiz Gallo]. https://hdl.handle.net/20.500.12893/7309

Jailani, J., Sugiman, S., \& Apino, E. (2017). Implementing the Problem-Based Learning in Order to Improve the Students' HOTS and Characters. Journal Riset Pendidikan Matematika, 4(2), 247-259. http://dx.doi.org/10.21831/jrpm.v4i2.17674

Leiva Sánchez, F. (2016). ABP como estrategia para desarrollar el pensamiento lógico matemático. Sophia 21. https://doi.org/10.17163/soph.n21.2016.09

Lestari, I., Nurmilawati, M., \& Santoso, A. (2015). Penerapan problem based learning (pbl) untuk meningkatkan kemampuan berpikir kritis dan sikap sosial peserta didik kelas VIII. The annual research report, 465-471. http://research-report.umm.ac.id/index.php/researchreport/article/view/487

Malmia, W., Hajiyanti Makatita, S., Lisaholit, S., A, A., Magfirah, I., Tinggapi, H., \& Basrun Umanailo, M. (2019). Problem Based Learning As An Effort To Improve Student Learning Outcomes. International Journal of Scientific \& Technology Research, 8(9), 1140-1143. https://doi.org/10.17163/soph.n21.2016.09

Ministerio de Educación. (2018). Evaluación PISA 2018. [Archivo PDF] http://umc.minedu.gob.pe/resultadospisa2018/

Ministerio de Educación. (2019). Evaluaciones de Logros de Aprendizaje. Oficina de Medición de la Calidad de los Aprendizajes. http://umc.minedu.gob.pe/minedu-publica-los-resultadosde-las-evaluaciones-nacionales-de-logros-de-aprendizaje-2019/ 
Mohd, M., Jawawi, R., Matzin, R., Shahrill, M., Jaidin, J., \& Mundia, L. (2016). The Benefits of Adopting a Problem-Based Learning Approach on Students' Learning Developments in Secondary Geography Lessons. International Education Studies, 9(2), 51-65 http://dx.doi.org/10.5539/ies.v9n2p51

Moreno Pinado, W., y Velázquez Tejeda, M. (2017). Estrategia Didáctica para Desarrollar el Pensamiento Crítico. Revista Iberoamericana sobre Calidad, Eficacia y Cambio en Educación, 15(2), 1-21. https://doi.org/10.15366/reice2017.15.2.003

Mulyanto, H., Gunarhadi, \& Indriayu, M. (2018). The Effect of Problem Based Learning Model on Student Mathematics Learning Outcomes Viewed from Critical Thinking Skills. International Journal of Educational Research Review, 3(2), 37-45. https://doi.org/10.24331/ijere.408454

Mundilarto, \& Helmiyanto, I. (2017). Effect of problem-based learning on improvement physics achievement and critical thinking of senior high school student. Journal of Baltic Science Education, 16 (5). 761-780.

Nasution, M., Yerizon, Y., \& Gusmiyanti, R. (5-6 de octubre de 2018). Students' Mathematical Problem-Solving Abilities Through The Application of Learning Models Problem Based Learning. [Discurso principal]. IOP Conference Series: Materials Science and Engineering, Padang, West Sumatera, Indonesia. https://doi.org/10.1088/1757-899X/335/1/012117

Neyra Quezada, E. (2020). Aprendizaje Basado en Problemas para el Aprendizaje significativo en Oatemática, en estudiantes de tercer año de secundaria, Chao 2019 [Tesis de doctorado, Universidad Cesar Vallejo]. https://hdl.handle.net/20.500.12692/44494

Nisa, R., Hussain, H., \& Anwar, N. (2017). Effects of Problem Based Learning on Students' Critical Thinking Skills, Attitudes towards Learning and Achievement. Journal of Educational Research, Dept. of Education, 20(2), 28-41.

Nuñez Huamanta, C. (2020). Aplicación de la estrategia ABP y su influencia en el desarrollo del pensamiento crítico en estudiantes de primaria - Mocan. [Tesis de doctorado, Universidad Nacional de Trujillo]. http://dspace.unitru.edu.pe/handle/UNITRU/15757

Rahamad, K., \& Hartono, D. (2016). Pengaruh model problem based learning (PBL) terhadap pemahaman konsep biologi dan keterampilan berpikir kritis siswa tentang ekosistem dan lingkungan di kelas X SMA Negeri 1 Sigi. Journal Sains dan Teknologi Tadulako, 5(1), 28-38.

Ramdiah, S., Abidinsyah, H., \& Mayasari, R. (2018). Problem-based learning: Generates higherorder thinking skills of tenth graders in ecosystem concept. Jurnal Pendidikan Biologi Indonesia, 4(1), 29-34. https://doi.org/10.22219/jpbi.v4i1.5490

Rodríguez Saenz, N. (2017). Aprendizaje basado en problemas en el desarrollo del pensamiento crítico y el rendimiento académico en Formación Ciudadana y Cívica, 2016. [Tesis de doctorado, Universidad Cesar Vallejo]. https://hdl.handle.net/20.500.12692/5338

Safitri, D., Irmawanty, Bachtiar, S., \& Yustika Rukman, Y. (2018). Students' cognitive achievement, critical thinking skills, and metacognitive awareness in problem based learning. European Journal of Education Studies, 5(4), 248-258. https://doi.org/10.5281/zenodo.1482095

Santyasa, W., Saindra, G., \& Juniantari, M. (agosto de 2019). Problem-based learning model versus direct instruction in achieving critical thinking ability viewed from students' social attitude in learning physics. [Discurso principal]. Proceedings of the 1st International Conference on Education Social Sciences and Humanities (ICESSHum 2019). https://doi.org/10.2991/icesshum-19.2019.101 
Sapallanay Baltazar, J. (2016). Estrategia del (ABP) y el desarrollo del Pensamiento crítico en estudiantes de cuarto y quinto de secundaria de la I.E. "Pueblo Libre" de la red educativa Mazamari - UGEL - Satipo-2016. [Tesis de doctorado, Universidad Católica Los Ángeles de Chimbote]. http://repositorio.uladech.edu.pe/handle/123456789/1406

Sastoque Gutiérrez, D., Ávila Palet, J., \& Olivares, S. (2016). Aprendizaje Basado en Problemas para la construcción de la competencia del Pensamiento Crítico. Voces y Silencios. Revista Latinoamericana de Educación, $\quad 7(1), \quad 148-172$. http://dx.doi.org/10.18175/VyS7.1.2016.08

Simamora, R., Rotua Sidabutar, D., \& Surya, E. (2017). Improving Learning Activity and Students' Problem Solving Skill through Problem Based Learning (PBL) in Junior High School. International Journal of Sciences: Basic and Applied Research (IJSBAR), 33(2), 321-331. https://www.researchgate.net/publication/317416532

Suryawati, E., Suzanti, F., Zulfarina, A., Putriana, R., \& Febrianti, L. (2020). The implementation of local environmental problembased learning student worksheets to strengthen environmental literacy. Jurnal Pendidikan IPA Indonesia, 9(2), 169-178. https://doi.org/10.15294/jpii.v9i2.22892

Tünnermann Bernheim, Carlos (2011). El constructivismo y el aprendizaje de los estudiantes. Universidades, $\quad$ (48), $21-32 . \quad$ ISSN: https://www.redalyc.org/articulo.oa?id=37319199005

UNESCO. (2017). La Agenda 2030 para el Desarrollo Sostenible. Organización de las Naciones Unidas para la Educación, la Ciencia y la Cultura, 1-22. [Archivo PDF] https://es.unesco.org/creativity/sites/creativity/files/247785sp_1_1_1.compressed.pdf

Urrútia, G., Bonfill, X. (2010). Declaración PRISMA: una propuesta para mejorar la publicación de las revisiones sistemáticas y metaanálisis. Medicina Clínica, 135 (11), 507-511. https://doi.org/10.1016/j.medcli.2010.01.015

Villalobos Delgado, V., Ávila Palet, J., \& Olivares, S. (2016). Aprendizaje basado en problemas en química y el pensamiento crítico en secundaria. Revista Mexicana de Investigación Educativa, 21(69), 557-581.

Wahyu, E., Sahyar, \& Ginting, E. (2017). The Effect of Problem Based Learning (PBL) Model toward Student's Critical Thinking and Problem Solving Ability in Senior High School. American Journal of Educational Research, 5(6), 633-638. https://doi.org/10.12691/education-5-6-7

Widyatiningtyas, R., Kusumah, Y., Sumarmo, U., \& Sabandar, J. (2015). The impact of problembased learning approach tosenior high school students' mathematics critical thinking ability. IndoMS-JME, 6(2), 107-116. https://doi.org/10.22342/jme.6.2.2165.107-116

Yanti, O., \& Indra, R. (2017). Model Problem based learning, guided inquiry, dan kemampuan berpikir kritis matematis. Jurnal Review Pembelajaran Matematika, 2(2), 120-130. https://doi.org/10.15642/jrpm.2017.2.2.120-130

Yuliati, L., Fauziah, R., \& Hidayat, A. (2018). Student's critical thinking skills in authentic problem based learning. Journal of Physics: Conference Series. 1013 (2018) 012025. https://doi.org/10.1088/1742-6596/1013/1/012025 